\title{
Diabetic ketoacidosis associated with atypical antipsychotic drug, clozapine treatment: Report of a case and review of literature
}

\author{
L. Pillai, S. M. K. Husainy, K. Ramchandani
}

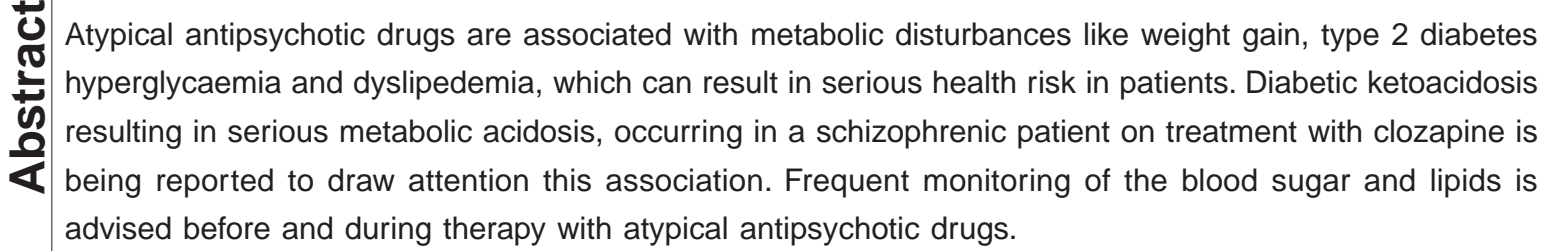

Key words: Atypical antipsychotics, clozapine, diabetic ketoacidosis lactic acidosis, respiridone

A close association between diabetes mellitus and psychiatric illness has been suggested in medical literature. ${ }^{[1-3]}$ In addition, type 2 diabetes, impaired glucose tolerance, exacerbation of preexisting type 1 and 2 diabetes mellitus and a severe, potentially fatal metabolic complications have been associated with antipsychotic drug treatment. ${ }^{[3-5]}$ A case report of a schizophrenic patient on atypical antipsychotic drugs who presented with severe metabolic acidosis is being presented to alert the critical care physicians about this association.

\section{Case Report}

A 45 years old male was transferred from a peripheral hospital where he was admitted unresponsiveness and in shock. He was a known case of schizophrenia for 10 years on various anti psychotic drugs. About 5 days before the admission he was treated by a general

\footnotetext{
From:

Department of Critical Care Medicine, Lokmanya Hospital, Chinchwad, Pune - 411033 , India
}

Correspondence:

Dr. Lalitha Pillai, Plot No. 82, Sector 26, Pradhikaran, Nigdi, Pune, India. E-mail: Ialithapillai@rediffmail.com practitioner for loose motion. He appeared to have recovered but on the day of admission he repeatedly complained of severe thirst, exhaustion and urinated very frequently. Gradually he became drowsy, progressively unresponsive and was taken to a hospital where they found that he had unrecoradable blood pressure. He was resuscitated with colloids and crystalloids and transferred here.

On arrival he appeared severely emaciated and was deeply unconscious with a respiratory rate of $8 / \mathrm{min}$ acidotic breathing and a BP of 60 systolic on inotropes. Pupils were bilaterally equal, reacting to light and there were no localizing neurological signs. He was immediately intubated and ventilated. Parental piperacillin-tazobactam was started, central line inserted, fluids and inotropes were titrated to optimize CVP and MAP. Arterial blood gas analysis showed pH of $6.9, \mathrm{PCO}_{2}$ $-32 \mathrm{~mm} \mathrm{Hg}, \mathrm{PO}_{2} 317 \mathrm{~mm} \mathrm{Hg}, \mathrm{HCO}_{3} 6.1 \mathrm{meq} / \mathrm{l}$, SBE 27. His hemoglobin was $6.4 \mathrm{gm} / \mathrm{dl}$, white cell count 25000/cmm, urea $102 \mathrm{mg} / \mathrm{dl}$, creatinine $2.8 \mathrm{mg} / \mathrm{dl}$, Na $125 \mathrm{meq} / \mathrm{l}$, K $3.1 \mathrm{mmeq} / \mathrm{l}, \mathrm{Cl} 90 \mathrm{meq} / \mathrm{l}$, S lactic acid $43 \mathrm{mg} / \mathrm{dl}$, HIV and HbsAg- Blood sugar was $416 \mathrm{mg} / \mathrm{dl}$ with glycosylated hemoglobin of $8.9 \mathrm{mg} \%$. Initial chest 
X-ray was normal, blood culture was later reported negative while, urine cultured E. coli $10^{5} \mathrm{cfu} / \mathrm{hpf}$ which was sensitive to the antibiotic used. Urine examination showed 10-15 pus cells/hpf and was positive for ketones and sugar. Drug levels of clonazepine and resperidone could not be done because the facility was not available but the possibility of drug overdose although considered but appeared unlikely as all the drugs were dispensed by the wife and were accounted for.

About 3 years back he was put on tab clozapine and resperidone. Around the same time he had been investigated for anaemia and all his reports including his blood sugar, renal and liver function tests were normal. Subsequently he had never been investigated. His mother had type 2 diabetes and had died of diabetes related complications. His elder and younger siblings had no diabetes. The patient was a tutor and depending on his clinical condition his wife adjusted his antipsychotic drug doses occasionally omitting them. Apparently he was on regular drugs for the last 3 months. In the recent days, the patient had a history of weight loss of more than $5 \mathrm{~kg}$.

For diabetic ketoacidosis with lactic acidosis as a result of septicemic shock, treatment was continued with insulin infusion, fluids, antibiotics and packed cell. By the end of $12 \mathrm{~h}$ the blood sugar was $200 \mathrm{mg} / \mathrm{dl}$ with normal electrolytes and absent ketones, but there was no improvement in the patients sensorium or blood pressure. He continued to have severe metabolic acidosis with ph 6.9 despite bicarbonate supplementation and remained hypotensive and died within 48 of admission.

\section{Discussion}

Literature search reveals a close association between psychiatric illness and diabetes..$^{[1-3,6-8]}$ Links between schizophrenia and diabetes have been reported even before the introduction of antipsychotic drugs. ${ }^{[3,5-7]}$ Poor dietary habits along with physical inactivity and poor motivation were amongst the reasons postulated for the increased incidence of diabetes mellitus. Introduction of the newer atypical antipsychotic drugs in the treatment of schizophrenia resulted in metabolic syndromes and further contributed to the occurrence of diabetes. ${ }^{[1-5,7,9,10]}$ Clozapine, olanzipine, risperidone and zotepine are important atypical antipsychotic drugs that were introduced to minimize the extra pyramidal side effects of the earlier drugs.

Metabolic profile caused by atypical anti psychotic drugs resembles type 2 diabetes. The contributory role of treatment induced weight gain has been indicated in many studies with clozapine and olanzipine having the greatest risk of developing significant weight gain while risperidone quetiapine, amisulpride and zotepine results in low to modest weight gain..$^{[4,5,7,9,10]}$ Because more than one quarter of the new cases of recent onset diabetes, had no weight gain and often diabetes was detected within a month of starting treatment with atypical antipsychotic drugs, it appears that these drugs may have direct action on glucose regulation, which is independent of adiposity. ${ }^{[3,4,7,10-12]}$ Patients have developed hyperglycaemia or deterioration when switched to risperidone or olanzapine after stopping clozapine. ${ }^{[7]}$ Other metabolic derangement involved lipid metabolism with clozapine been linked to hypertriglyceridemia and risperidone with reduction in plasma triglycerides. ${ }^{[3,5]}$ Risk factors for developing diabetes were overweight,weight gain during treatment, age, ethnicity, family or personal history of diabetes mellitus or hypertension ${ }^{[1]}$ Newcomer et al. ${ }^{[13]}$ noted that although $50 \%$ of the practicing psychiatrists were aware of the metabolic consequences of these drugs such as diabetes and weight gain, only $2 \%$ were aware of complications like ketoacidosis. ${ }^{[13]}$

Postulated mechanisms responsible for the development of diabetes by these drugs include, increased insulin resistance, their effect on serotonin receptors and weight gain through antagonism at receptors sites..$^{[1,3]}$ Lean et al..[4] found patients younger than 40 years on atypical antipsychotic drugs to have an odds ratio of $1.6(95 \% \mathrm{Cl} 1.23-2.16)$ to develop type 2 diabetes. ${ }^{[4]}$ Jin and collogues ${ }^{[8]}$ analyzed 45 published cases of new onset diabetes associated with atypical antipsychotic therapy. Their mean age was 40.3 years at detection and $50 \%$ of them had no weight gain. The mean duration of drug treatment before onset of diabetes in this study was 19 weeks (range 2-124 weeks). They also concluded that weight gain during treatment did not appear to be a major risk for development of diabetes.

Koller et al. studied 384 cases of clozapine-associated diabetes reported between 1990 and 2001. [7] Like in the earlier series $40 \%$ of the cases presented in the fourth decade of life. Diabetes was diagnosed in $27 \%$ in the 
first month and in 56\% within the first 3 months of clozapine therapy with occurrence as early two days in case of an accidental ingestion and as late as 5 years. This survey showed no association between the doses and severity of hyperglycaemia, presence of acidosis or time to onset of hyperglycaemia. In most of the cases diabetes improved on discontinuation of clozapine,

Diabetic ketoacidosis (DKA) is a serious acute medical condition. The fact that DKA can occur as a first presentation is alarming as it has considerable morbidity and mortality. In Jin et al. series, $42 \%$ of the recent onset diabetes presented with DKA directly while in Koller and collogues survey of the 80 cases with metabolic acidosis or ketoacidosis, $91 \%$ were new onset diabetics. In the same study of the 25 patients who died, 16 patients had ketosis but most of them had associated medical problems, which made it difficult like in our case, to determine whether ketoacidosis was the primary or additional cause of death. Severe metabolic acidosis in our case was due to both diabetic ketoacidosis and lactic acidosis, which we felt, was explained by the presence of septic shock but, severe lactic acidosis with fatal myocardial infarction due to clozapine has been reported by Koren et al..[14]

Our patient also was at the age of 40 , with a family history of diabetes a known risk factor for drug induced diabetes. He presented with altered sensorium and in ketoacidosis. Clozapine induced sedation has been reported to contribute to the delay in diagnosis of diabetes. ${ }^{[7]}$ Changes in mental status, was reported in 11 out of the 45 cases of recent onset severe hyperglycaemia in Koller et al. series. ${ }^{[7]}$ Although he was on therapy with clozapine for 3 years, there were periods when he was of off drugs. These drug free periods may have reversed any hyperglycaemia occurring during the drug period or contributed to the delay in onset of diabetes. During the last 3 months he was on continuous treatment with two potent diabetes inducing drugs, clozapine and resperidone. Raised glycosylated levels prove that his blood sugars were high over the last 2-3 months. Although type 2 diabetes is associated with clozapine, diabetic ketoacidosis probably represents a severe form of the metabolic disturbance with total exhaustion of the pancreatic beta cells to produce insulin. ${ }^{[8]}$

The inadvertent unsupervised self-medication by the patient and family resulted in the patient not being regular on follow up. Patients on long-term atypical antipsychotic drugs should undergo regular monitoring of plasma sugar and lipids. ${ }^{[5,6]}$ The care givers and family members should also be instructed on the signs and symptoms of diabetes, side effect of the drugs and the need for regular blood tests. Physicians caring for the patient will need to modify or omit these drugs to prevent relapse.

\section{Conclusion}

Clozapine and resperidone have been reported to result in nondose dependent hyperglycemia, which is reversible on stopping the treatment, reappearing on reintroduction of these drugs. Knowledge of this association is important for critical care physicians and treating psychiatrists to avoid inadvertent reintroduction of the drugs. Patients need to have their lipids and sugar checked regularly to avoid catastrophic complications.

\section{References}

1. Lindenmayer JP, Nathan AM, Smith RC. Hyperglycemia associated with the use of atypical antipsychotics. J Clin Psychiatr $2001 ; 62: 30-8$.

2. Krosnick $A$, Wilson MG. A retrospective chart review of the clinical effects of atypical antipsychotic drugs on glycemic control in institutionalized patients with schizophrenia and comorbid diabetes mellitus. Clin Ther 2005;27:320-6.

3. Haupt DW, Newcomer JW. Hyperglycemia and antipsychotic medication. J Clin Psychiatr 2001;62:15-26.

4. Lean ME, Pajonk FG. Patients on atypical antipsychotic drugs: Another high-risk group for type 2 diabetes. Diabetes Care 2003;26:1597-605.

5. Newcomer JW. Second-generation (atypical) antipsychotics and metabolic effects: A comprehensive literature review. CNS Drugs 2005;19:1-93.

6. Bergman RN, Ader M. Atypical antipsychotics and glucose heomeostasis. J Clin Psychiatr 2005;66:504-14.

7. Koller E, Schneider B, Bennett K, Dubitsky G. Clozapine associated diabetes. Am J Med 2001;111:716-23.

8. Jin H, Meyer JM, Jeste DV. Phenomenology of and risk factors for new onset diabetes mellitus and diabetic ketoacidosis associated with atypical antipsychotics: An analysis of 45 published cases. Ann Clin Psychiatr 2002;14:59-64.

9. Newcomer JW. Metabolic risk during antipsychotic treatment. Clin Ther 2004;26:1936-46.

10. Ananth J, Venkatesh R, Burgoyne K, Gunatilake S. Atypical antipsychotic drug use and diabetes. Psychother Psychosom 2002;71:244-54. 
11. Henderson DC, Cagliero E, Gray C, Nasrallah RA, Hayden DL, Schoenfeld DA, et al. Clozapine, diabetes mellitus, weight gain and lipid abnormalities: A five- year naturalistic study. Am J Psychiatr 2000;157:975-81

12. Haggs S, Joelsson L, Mjorndal T, Spigset O, Oja G, Dahlqvist R. Prevalence of diabetes and impaired glucose tolerance in patients treated with clozapine compared with patients treated with conventional depot neuroleptic medications. J Clin Psychiatr 1998;59:294-9.

13. Newcomer JW, Nasrallah HA, Loebel AD. The atypical antipsychotic therapy and metabolic issues national survey: Practice patterns and knowledge of psychiatrists. J Clin Psychopharmacol 2004;24:S1-6.

14. Koren W, Kresis Y, Duchowiczny K, Prince T, Sancovici S, Sidi Y, et al. Lactic acidosis and fatal myocardial infarction due to clozapine. Ann Pharmacother 1997;31:168-70.

Source of Support: Nil, Conflict of Interest: None declared. 